\title{
Editorial
}

\section{Concepts of Novel Nanomaterial Device and Application}

\author{
Ting-Jen Hsueh, ${ }^{1}$ Sheng-Po Chang, ${ }^{2}$ Shyh-Jer Huang, ${ }^{3}$ \\ Chieh-Chih Huang, ${ }^{4}$ and Michael $\mathrm{Hu}^{5}$ \\ ${ }^{1}$ National Nano Device Laboratories, Tainan-Based Science Park, Tainan 741, Taiwan \\ ${ }^{2}$ Advanced Optoelectronic Technology Center, National Cheng Kung University, Tainan 70101, Taiwan \\ ${ }^{3}$ Department of Electrical Engineering, University of California, Los Angeles, CA 90095-1594, USA \\ ${ }^{4}$ Low Energy Electronic Systems, Singapore-MIT Alliance for Research and Technology, Singapore 138602 \\ ${ }^{5}$ Oak Ridge National Laboratory, Oak Ridge, TN 37831, USA
}

Correspondence should be addressed to Ting-Jen Hsueh; tj.hsueh@gmail.com

Received 23 June 2014; Accepted 23 June 2014; Published 10 July 2014

Copyright (C) 2014 Ting-Jen Hsueh et al. This is an open access article distributed under the Creative Commons Attribution License, which permits unrestricted use, distribution, and reproduction in any medium, provided the original work is properly cited.

Nanomaterial comprises several hundred atoms and molecules, which a lot of complicated and interesting interactions occur in it. Characteristics in nanoscale, such as transmittance, electrical conductivity, thermal conductivity, magnetic, corrosion, and other effects.

Furthermore, nanomaterial manufacturing technology is applied on all kinds of nanomaterial devices, such as photo device, FET, and biosensor, and enhances electronic, optical, and mechanical properties. Most importantly, taking advantage of nanotechnology, the cost of devices will go down with better quality.

This special issue is about novel nanomaterials device and applications and other related fields. This special issue enables interdisciplinary collaboration between material science and engineering technologists in the academic and industrial fields. The paper describes sealed and nonsealed $\mathrm{InGaP} / \mathrm{InGaAs} / \mathrm{Ge}$ triple-junction solar cell in a complex high temperature and high humidity environment and investigated the electrical properties over time. Another paper presents combined two-dimensional-threedimensional (2D-3D) ensemble Monte Carlo (EMC) model, and the performance of a planar nanochannel device is studied at the terahertz $(\mathrm{THz})$ region. Another paper is on $\mathrm{Mo} / \mathrm{Si}$ bilayer thin films which were grown by magnetron sputtering and applied to write-once blu-ray disc (BD-R). Another paper studies the ZnS thin films which were prepared on glass substrates by chemical bath deposition at various $\mathrm{Zn} / \mathrm{S}$ molar ratios from $1 / 50$ to $1 / 150$. Another paper describes low-frequency noise characterizations in $28 \mathrm{~nm}$ high-k (HK)
pMOSFET with embedded SiGe source/drain (S/D) through noise and random telegraph noise measurements simultaneously. Another paper adopts transparent electrodes of tin dioxide $\left(\mathrm{SnO}_{2}\right)$ on glasses which were further wet-etched in the diluted $\mathrm{HCl}: \mathrm{Cr}$ solution to obtain larger surface roughness and better light-scattering characteristic for thinfilm solar cell applications.

The paper describes A $20 \mathrm{~nm} \mathrm{SiO}_{x}$ layer which is deposited using radiofrequency sputtering to form the resistive switching layer of a $\mathrm{Cu} / \mathrm{SiO}_{x} / \mathrm{Pt}$ memory device. Another paper proposes $\mathrm{Mg}_{0.1} \mathrm{Zn}_{0.9} \mathrm{O}$ films which were grown using atomic layer deposition (ALD) system and applied to metal-semiconductor-metal ultraviolet photodetectors (MSM-UPDs) as an active layer. Another paper describes GaN-based flip-chip white light-emitting diodes (FCWLEDs) with diffused $\mathrm{ZnO}$ nanorod reflector and with $\mathrm{ZnO}$ nanorod antireflection layer which were fabricated. Another paper proposes to use silicon nanoparticles doped poly(3-hexylthiophene) and [6,6]-phenyl C61-butyric acid methyl ester blends (P3HT: PCBM : Si NP) which have been produced as the photoactive layer of organic photovoltaic devices (OPVs). Another paper of this special issue uses magnesium-nickel $(\mathrm{Mg}-\mathrm{Ni})$ powders which are used as the anode materials for secondary lithium (Li) ion batteries.

Ting-Jen Hsueh Sheng-Po Chang Shyh-Jer Huang Chieh-Chih Huang Michael $\mathrm{Hu}$ 

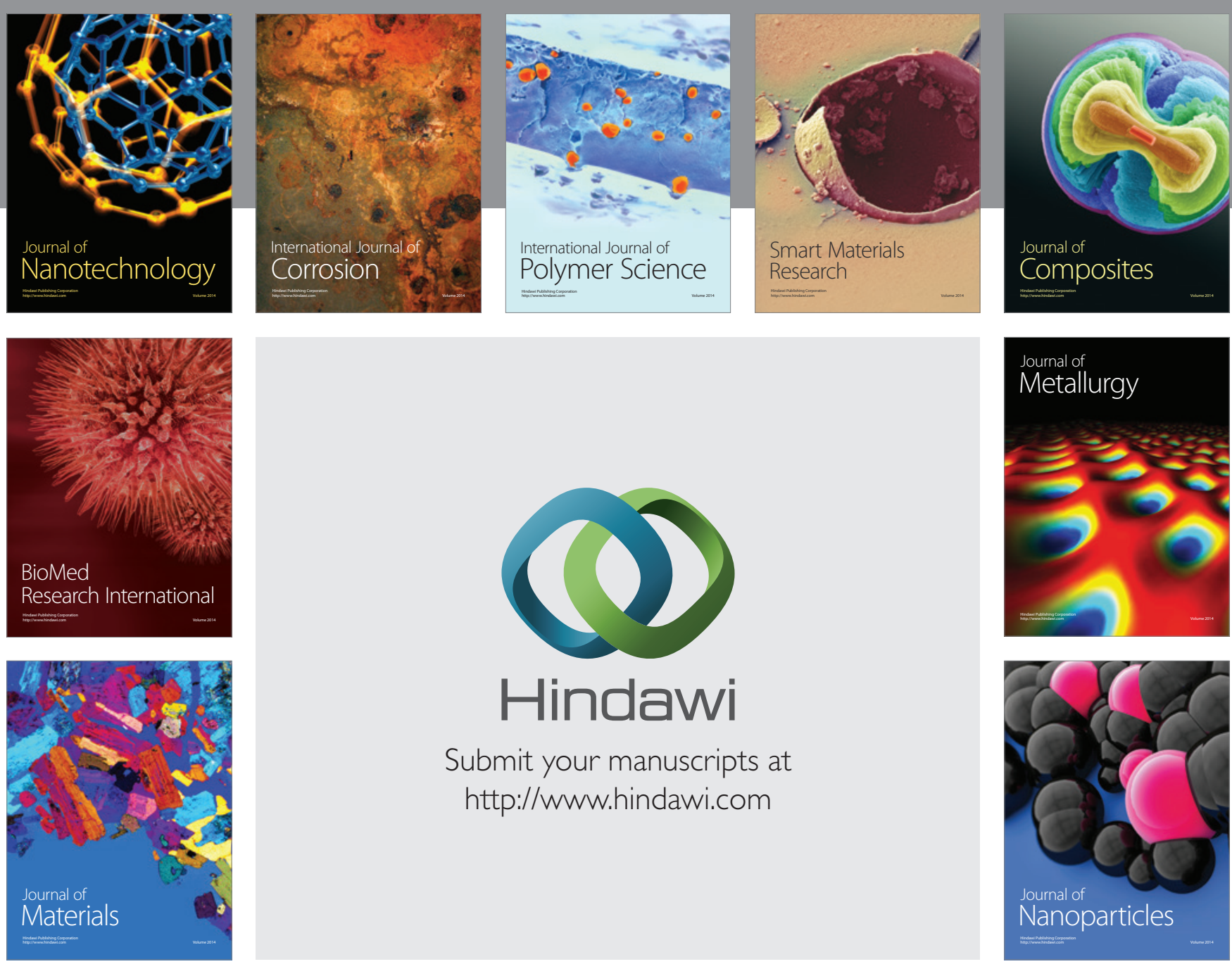

Submit your manuscripts at http://www.hindawi.com
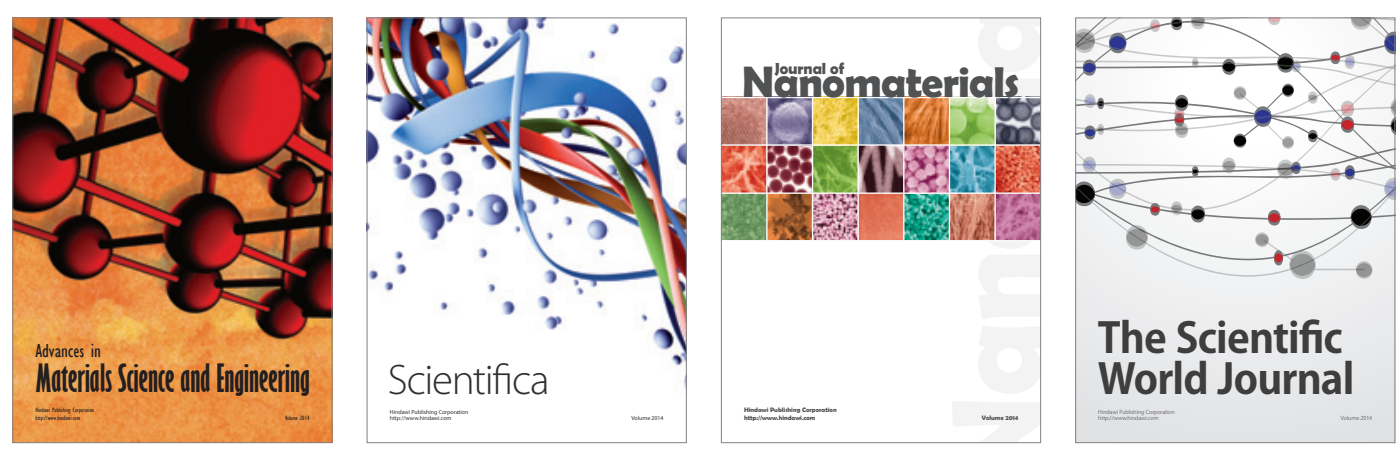

\section{The Scientific World Journal}
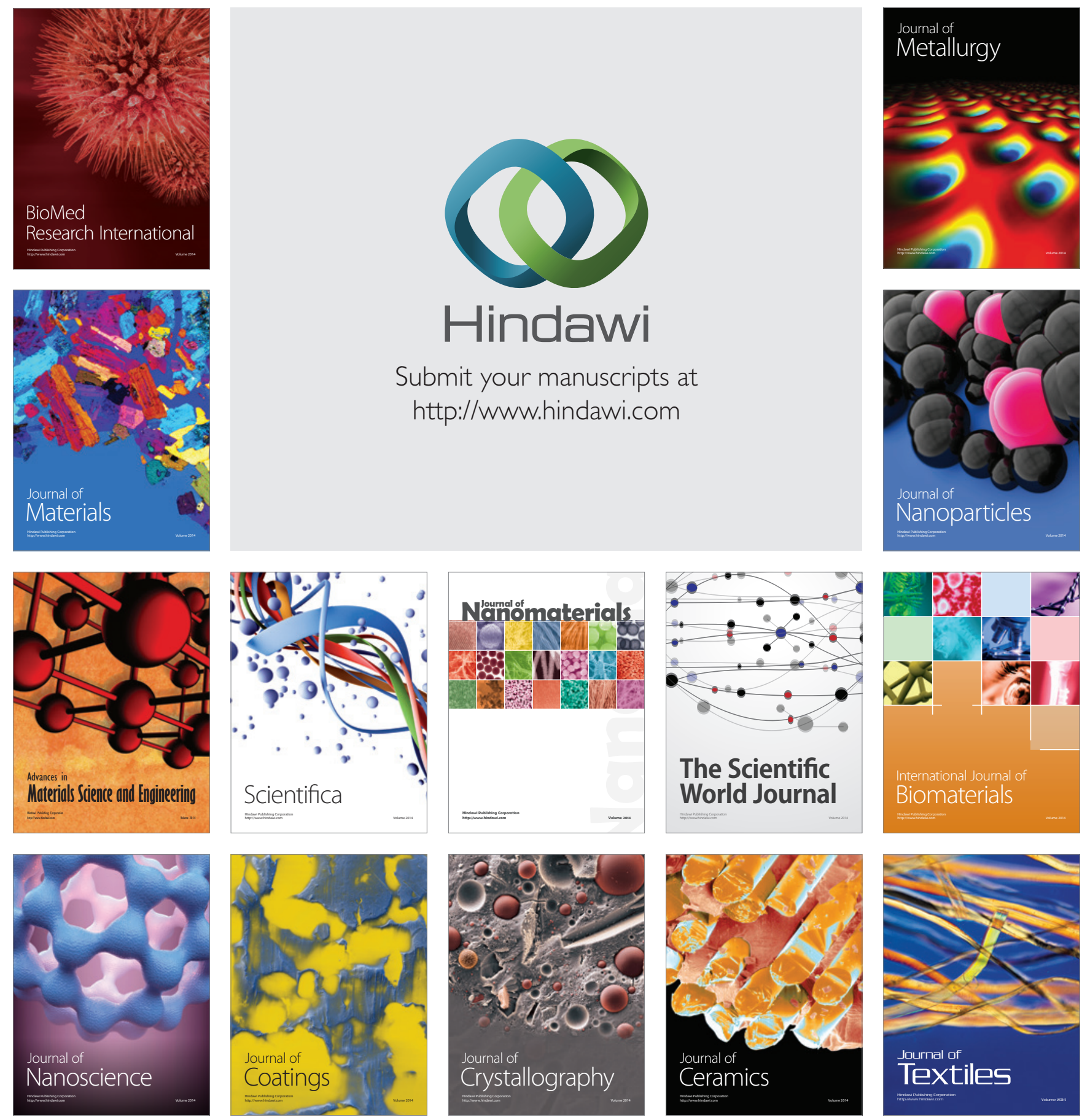